\title{
Correction to: Cross-cultural and hemispheric laterality effects on the ensemble coding of emotion in facial crowds
}

\author{
Hee Yeon $\mathrm{Im}^{1} \cdot$ Sang Chul Chong ${ }^{2,3} \cdot$ Jisoo Sun $^{2}$ • Troy G. Steiner ${ }^{4}$. \\ Daniel N. Albohn ${ }^{4} \cdot$ Reginald B. Adams Jr. ${ }^{4}$ Kestutis Kveraga ${ }^{1}$
}

\section{Correction to: Cult. Brain (2017) 5:125-152 https://doi.org/10.1007/s40167-017-0054-y}

In the original publication of the article under Introduction, the sentence in the second paragraph that reads as, "Therefore, none of the existing studies...Westerners" should read as, "Therefore, none of the existing studies on crowd emotion perception allows us to directly compare potential differences between Easterners and Westerners in extracting crowd emotion from groups of faces of Easterners vs Westerners."

\footnotetext{
The original article can be found online at https://doi.org/10.1007/s40167-017-0054-y.

Kestutis Kveraga

kestas@nmr.mgh.harvard.edu

Sang Chul Chong

scchong@yonsei.ac.kr

Reginald B. Adams Jr.

regadams@psu.edu

1 Department of Radiology, Massachusetts General Hospital, Athinoula A. Martinos Center for Biomedical Imaging, Harvard Medical School, Charlestown, MA 02129, USA

2 Graduate Program in Cognitive Science, Yonsei University, Seoul, South Korea

3 Department of Psychology, Yonsei University, Seoul, South Korea

4 Department of Psychology, The Pennsylvania State University, State College, PA, USA
} 